\title{
Controlled Release of Stromal-Cell- Derived Factor-1 from Gelatin Hydrogels Enhances Angiogenesis
}

\section{$\operatorname{AUTHOR}(\mathrm{S})$ :}

Kimura, Yu; Tabata, Yasuhiko

\section{CITATION:}

Kimura, Yu ...[et al]. Controlled Release of Stromal-Cell-Derived Factor-1 from Gelatin Hydrogels Enhances Angiogenesis. Journal of Biomaterials Science, Polymer Edition 2010, 21(1):37-51

ISSUE DATE:

2010

URL:

http://hdl.handle.net/2433/148424

RIGHT:

(c) Koninklijke Brill NV, Leiden, 2010 


\title{
Controlled Release of Stromal-Cell-Derived Factor-1 from Gelatin Hydrogels Enhances Angiogenesis
}

\author{
Yu Kimura and Yasuhiko Tabata* \\ Department of Biomaterials, Field of Tissue Engineering, Institute for Frontier Medical Sciences, \\ Kyoto University, 53 Kawara-cho Shogoin, Sakyo-ku, Kyoto, 6068507, Japan
}

Received 14 November 2008; accepted 22 December 2008

\begin{abstract}
Controlled release of a chemokine, stromal-cell-derived factor-1 (SDF-1), could be achieved with gelatin hydrogels of release carrier. Gelatin was chemically derivatized to give it different electric charge and hydrophobicity. Among the derivatives, succinylated gelatin (Succ) of an anionic charge was the most suitable for preparation of the hydrogel in terms of SDF-1 release. The time profile of SDF-1 release from the hydrogel of succinylated gelatin could be controlled by changing the water content of hydrogel which could be modified by changing the conditions of hydrogel preparation. When evaluated after the subcutaneous implantation of Succ hydrogels incorporating SDF-1 or injection of SDF-1 solution, significantly stronger angiogenesis by the hydrogel was observed. The hydrogel implantation also enhanced the mRNA level of SDF-1 receptor at the site implanted. It is possible that the gelatin hydrogel enabled SDF-1 to be released locally, resulting in an enhanced angiogenesis at the site implanted.

(C) Koninklijke Brill NV, Leiden, 2010
\end{abstract}

Keywords

Stromal-cell-derived factor-1 (SDF-1), controlled release, angiogenesis, gelatin hydrogel

\section{Introduction}

As a new strategy to treat defective tissues, the therapy of regenerative medicine by tissue engineering has been expected. In the concept of tissue engineering, cells and the local environment, which is composed of scaffolds and bioactive molecules, are combined to use for the natural induction of tissue regeneration [1]. We have explored biodegradable gelatin hydrogels for the controlled release of bioactive molecules, such as growth factors and plasmid DNAs, to enhance their in vivo biological activities [2]. This release system is one of the drug-delivery system technologies to prolong their in vivo half-life period and consequently enhance the therapeutic efficacy at reduced injection doses. Some clinical trials of angiogene-

\footnotetext{
* To whom correspondence should be addressed. Tel.: (81-75) 751-4121; Fax: (81-75) 751-4646; e-mail: yasuhiko@frontier.kyoto-u.ac.jp 
sis therapy by the release technology of basic fibroblast growth factor (bFGF) have been started in Japan to demonstrate the therapeutic feasibility [3].

A chemokine, stromal-cell-derived growth factor (SDF-1, CXCL12), is known as a chemokine which functions in the inflammation reaction, leukocyte development, and the maintenance and recruitment of stem cells [4-7]. Additionally, SDF-1 has an inherent ability to mobilize hematopoietic stem cells with the receptor CXCR4 from the bone marrow [8].

In this study, the hydrogel release technology was applied to SDF-1 and the biological potential was evaluated in terms of SDF-1-induced angiogenesis. Hydrogels were prepared from various gelatin derivatives with different physicochemical properties to achieve the controlled release of SDF-1. Following the subcutaneous implantation of gelatin hydrogels incorporating SDF-1 into the back of mice, angiogenesis was evaluated and compared with that of SDF-1 solution injection. We examined the in vivo profiles of SDF-1 release from gelatin hydrogels with different biodegradabilities.

\section{Materials and Methods}

\subsection{Materials}

Gelatin samples, prepared by an alkaline treatment of bovine bone collagen (isoelectric point $($ IEP $)=5.0$ ) and an acidic treatment of pig skin collagen $($ IEP $=9.0)$, termed as the acidic type and basic type here, were kindly supplied by Nitta Gelatin (Osaka, Japan). Recombinant human stromal-cell-derived factor-1 (SDF-1, 350-NS/CF) was obtained from R\&D systems (Minneapolis, MN, USA). Na ${ }^{125} \mathrm{I}$ (NEZ-033H, > 12.95 GBq/ml) and $\mathrm{N}^{\prime}$-succinimidyl-3-(4-hydroxy-3,5-di[ $\left.{ }^{125} \mathrm{I}\right]$ iodophenyl)propionate ([ $\left.{ }^{125} \mathrm{I}\right]$ Bolton-Hunter reagent, NEX-120H, $147 \mathrm{MBq} / \mathrm{ml}$ ) were purchased from Perkin-Elmer Life Sciences (Boston, MA, USA). Glutaraldehyde (GA), glycine, and other chemicals were obtained from Wako (Osaka, Japan) and used without further purification.

\subsection{Preparation of Gelatin Derivatives with Various Isoelectric Points}

Gelatin was chemically derivatized with succinic anhydride (Succ), decylamine (C10), spermine (SM) and ethylenediamine (ED) with or without 1-ethyl-3-(3dimethylaminopropyl)carbodiimide hydrochloride (EDC) of a catalyst to change the electronic nature as previously described [9]. The IEP of derivatives was measured according to the method previously reported [10]. Briefly, $1 \mathrm{wt} \%$ of gelatin derivative solution was applied for an ion exchange column packed with mixed cationic (Dowex 50W-X8) and anionic (Dowex 1-X8) exchange resins at $40^{\circ} \mathrm{C}$ and the $\mathrm{pH}$ of elution solution was measured with a $\mathrm{pH}$ meter (D-22, Horiba, Kyoto, Japan) at $40^{\circ} \mathrm{C}$. 


\subsection{Preparation of Gelatin Hydrogels}

An aqueous solution of $5 \mathrm{wt} \%$ each gelatin derivative was mixed with various amounts of glutaraldehyde, followed by leaving at $4{ }^{\circ} \mathrm{C}$ for $12 \mathrm{~h}$ for gelatin crosslinking. The cross-linked gelatin hydrogel was treated by $0.1 \mathrm{M}$ glycine solution to block the residual aldehyde groups. After washing with double-distilled water (DDW) three times, the hydrogels were freeze-dried. The cross-linking extent of hydrogels prepared was evaluated by measuring the water content according to the method previously described [11].

\subsection{In Vitro Release Test of SDF-1 from Gelatin Hydrogels}

SDF-1 was radioiodinated through the conventional chloramine-T method as previously described [12]. Briefly, $5 \mu \mathrm{l}$ of $\mathrm{Na}^{125}$ I was added to $200 \mu \mathrm{l}$ of SDF-1 solution in $0.5 \mathrm{M}$ potassium phosphate buffer $(\mathrm{pH} 7.5)$ containing $0.5 \mathrm{M} \mathrm{NaCl}$. Then, $0.2 \mathrm{mg} / \mathrm{ml}$ chloramine-T in the same buffer $(100 \mu \mathrm{l})$ was added to the solution mixture. After agitation at room temperature for $2 \mathrm{~min}, 100 \mu \mathrm{l}$ of phosphate-buffered saline (PBS) solution ( $\mathrm{pH} 7.5$ ) containing $0.4 \mathrm{mg}$ sodium metabisulfate was added to the reaction solution to stop the radioiodination. The reaction mixture was passed through a PD-10 desalting column (GE Healthcare Life Sciences, Chalfont St Giles, UK) to remove the uncoupled, free ${ }^{125}$ I molecules from the ${ }^{125}$ I-labeled SDF-1. A PBS solution of ${ }^{125}$ I-labeled SDF-1 $(20 \mu \mathrm{l})$ was dropped onto the freeze-dried hydrogel of gelatin derivatives $\left(2 \mathrm{mg}, 2.5 \times 2.5 \times 3 \mathrm{~mm}^{3}\right)$, followed by leaving at $25^{\circ} \mathrm{C}$ for $3 \mathrm{~h}$ to obtain hydrogels incorporating ${ }^{125} \mathrm{I}$-labeled SDF-1. For the in vitro release test, one hydrogel incorporating ${ }^{125} \mathrm{I}$-labeled SDF-1 was agitated at $37^{\circ} \mathrm{C}$ in $1 \mathrm{ml}$ PBS. The supernatant was removed $0.5,1,2,4,8$ and $24 \mathrm{~h}$ later and replaced with the same volume of fresh PBS. The radioactivity of each supernatant was measured on a gamma counter (ARC-301B, Aloka, Tokyo, Japan) to evaluate the time profile of SDF-1 release ( $n=3$ at each time point).

\subsection{In Vivo Release Test of SDF-1 from Gelatin Hydrogels}

All the animal experiments were performed according to the Institutional Guidance of Kyoto University on Animal Experimentation and under permission by animal experiment committee of Institute for Frontier Medical Science, Kyoto University. All the surgical and observation procedures were performed under continuous inhalation anesthesia by isoflurane (Forane ${ }^{\circledR}$, Abbott Japan, Osaka, Japan) with 400 units of anesthesia (Univentor, Zejtun, Malta). PBS containing ${ }^{125}$ I-labeled SDF-1 $(20 \mu \mathrm{l})$ was dropped onto $2 \mathrm{mg}$ of freeze-dried gelatin hydrogels, followed by incubation at $25^{\circ} \mathrm{C}$ for $3 \mathrm{~h}$ to allow to swell into the hydrogel. Following the implantation of gelatin hydrogels incorporating ${ }^{125}$ I-labeled SDF-1 into the back subcutis of female ddY mice, 6 weeks old (18-20 g body weight, Shimizu Laboratory Supply, Kyoto, Japan), tissue around the site implanted was extracted at different time intervals after hydrogel implantation, and the tissue radioactivity was counted by the gamma counter to estimate the in vivo time profiles of SDF-1 release ( $n=3$ at each time point). 


\subsection{In Vivo Evaluation of Gelatin Hydrogel Degradation}

To evaluate the degradation profiles of gelatin hydrogels, the in vivo implantation of ${ }^{125} \mathrm{I}$-labeled hydrogels was performed according to the method previously reported $[12,13]$. Briefly, $\left[{ }^{125} \mathrm{I}\right]$ Bolton-Hunter reagent solution $(20 \mu \mathrm{l})$ in benzene was completely evaporated under dry nitrogen. The resultant reagent was dissolved into $1 \mathrm{ml}$ of PBS ( $\mathrm{pH} 7.5$ ). The reagent solution was impregnated into the gelatin hydrogel sheet $\left(2.5 \times 2.5 \times 3 \mathrm{~mm}^{3}\right)$ at a volume of $20 \mu \mathrm{l}$ per sheet. The sheets were incubated at $4{ }^{\circ} \mathrm{C}$ for $3 \mathrm{~h}$ to introduce ${ }^{125} \mathrm{I}$ into the amino groups of gelatin. The radioiodinated sheets were washed with DDW thoroughly $\left(4^{\circ} \mathrm{C}, 4\right.$ days) to exclude non-reacted ${ }^{125}$ I reagent, till the radioactivity of DDW returned to a background level. ${ }^{125}$ I-labeled gelatin hydrogels were implanted into the back subcutis of mice. Then, tissue around the sites implanted was extracted at different time intervals after hydrogel implantation, and the tissue radioactivity was counted by the gamma counter to obtain the degradation profiles of hydrogels over time $(n=3$ at each time point).

\subsection{RT-PCR Measurement}

After implantation of SDF-1-incorporated gelatin hydrogels into the back subcutis, $15 \times 15 \mathrm{~mm}^{2}$ of skin tissue around the site implanted was taken out with a surgical scalpel. The total RNA was extracted by using RNeasy fibrous tissue mini kit (Qiagen, Valencia, CA, USA) according to the manufacturers' instructions. Reverse transcription reaction was performed with the SuperScript II First-Stand Synthesis System (Invitrogen, Carlsbad, CA, USA). Real-time PCR was performed on a Prism 7500 real time PCR thermal cycler (Applied Biosystems, Foster City, CA, USA) from $10 \mathrm{ng}$ of cDNA in a total volume of $25 \mu$ l containing Power SYBR Green PCR Master Mix (Applied Biosystems) and $10 \mu \mathrm{M}$ of each primer (Table 1). The reaction mixture was incubated for the initial denaturation at $95^{\circ} \mathrm{C}$ for $10 \mathrm{~min}$, followed by 40 PCR cycles. Each cycle consisted of the following three steps; $94^{\circ} \mathrm{C}$ for $15 \mathrm{~s}, 57^{\circ} \mathrm{C}$ for $15 \mathrm{~s}$ and $72^{\circ} \mathrm{C}$ for $1 \mathrm{~min}$. Each mRNA level was normalized by the expression level of $18 \mathrm{~S}$ ribosomal RNA as an internal control.

Table 1.

Primer sequences used for real time PCR

\begin{tabular}{lll}
\hline Name & Primer & \\
\hline CXCR4 & Sense & GCTGGCTGAAAAGGCAGTCTAT \\
& Antisense & TGACGTCGGCAAAGATGAAGT \\
$18 \mathrm{~S}$ & Sense & ACTCAACACGGGAAACCTCA \\
& Antisense & AACCAGACAAATCGCTCCAC \\
\hline
\end{tabular}




\subsection{Angiogenesis Assay of Gelatin Hydrogels Incorporating SDF-1}

The biological activity of SDF-1 incorporated in gelatin hydrogels was evaluated by using a skinfold chamber attached on the back skin of mice, according to the study by Ichioka et al. [14]. The gelatin hydrogel incorporating $5 \mu \mathrm{g}$ of SDF-1 was implanted into the skin defect in the chamber 3 days after the chamber attachment. The number of capillaries newly-formed around the hydrogel implanted was microscopically counted at different time intervals using a Stemi 2000C stereomicroscope (Carl Zeiss Japan, Tokyo, Japan).

\subsection{Statistical Analysis}

All the results were expressed as the mean \pm standard deviation. For statistical analysis, the Tukey-Kramer post-test for multiple comparisons was used and differences were considered to be significant at $P<0.05$.

\section{Results}

\subsection{Characterization of Gelatin Derivatives and Their Hydrogels}

Table 2 shows the isoelectric point and degree of introduction of gelatin derivatives prepared, and the water content of their hydrogels cross-linked with glutaraldehyde. pI5 and pI9 gelatin were alkaline and acid-processed gelatin raw materials. Thus, the degree of introduction was not indicated. Succinylation of gelatin decreased the IEP while the derivatization with spermine and ethylenediamine increased it. Chemical derivatization modified the electric charge of gelatin. For in vitro and in vivo release experiment, hydrogels from gelatin derivatives with water contents of $97-98 \%$ were used. Table 3 shows the water content of hydrogels prepared from succinylated gelatin. The water content was changed by altering the GA concentration in hydrogel preparation.

\section{Table 2.}

Isoelectric points and degree of introduction of gelatin derivatives used for hydrogel preparation, and water contents of the hydrogels cross-linked with glutaraldehyde

\begin{tabular}{lcccccl}
\hline & Succ & C10 & pI5 & pI9 & SM & ED \\
\hline IEP & 4.57 & 4.72 & 5.09 & 8.90 & 10.83 & 11.21 \\
$\begin{array}{l}\text { Degree of } \\
\text { introduction } \\
\quad(\text { mol/mol\%) }\end{array}$ & $29.0 \pm 1.2$ & $28.6 \pm 2.4$ & - & - & $49.0 \pm 1.1$ & $50.9 \pm 1.1$ \\
$\begin{array}{l}\text { The water content } \\
\text { of hydrogels } \\
\text { (wt\%) }\end{array}$ & $97.9 \pm 0.0$ & $97.4 \pm 0.2$ & $97.8 \pm 0.2$ & $98.2 \pm 0.1$ & $97.4 \pm 0.8$ & $97.9 \pm 0.6$ \\
\hline
\end{tabular}

Succ, succinylated gelatin; C10, decylamine-introduced gelatin; $\mathrm{pI}$, acidic type gelatin; $\mathrm{pI}$, basic type gelatin; SM, spermine-introduced gelatin; ED, ethylenediamine-introduced gelatin. 
Table 3.

Preparation conditions and water content of succinylated gelatin hydrogels

\begin{tabular}{lll}
\hline Gelatin (wt\%) & Glutaraldehyde $(\mathrm{mM})$ & Water content $(\mathrm{wt} \%)$ \\
\hline 5 & 44.9 & $95.8 \pm 0.1$ \\
5 & 22.4 & $96.3 \pm 0.1$ \\
5 & 11.2 & $97.9 \pm 0.0$ \\
5 & 6.24 & $98.8 \pm 0.1$ \\
\hline
\end{tabular}

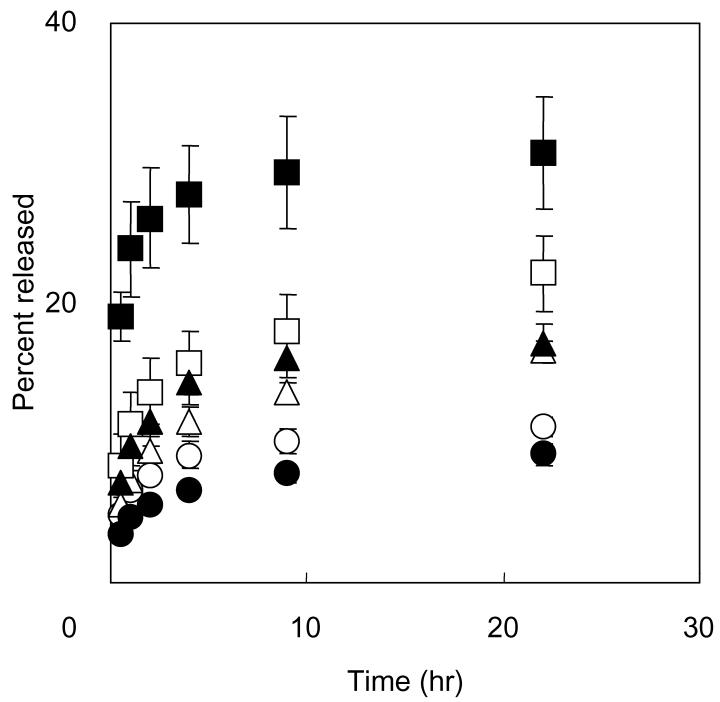

Figure 1. In vitro release profiles of SDF-1 from hydrogels of gelatin-derivatives; succinylated gelatin $(\operatorname{Succ})(O)$, decylamine-introduced gelatin $(\mathrm{C} 10)(\triangle)$, acidic type gelatin (pI5) $(\square)$, basic type gelatin $($ pI9) $(\bullet)$, spermine-introduced gelatin $(\mathrm{SM})(\mathbf{\Lambda})$ and ethylenediamine-introduced gelatin (ED) (ם).

Figure 1 shows the time profile of SDF-1 release from various gelatin hydrogels. The release profiles depended on the type of gelatin hydrogels. Irrespective of the hydrogel type, the amount of SDF-1 released from the gelatin hydrogels reached to a certain level and saturated. Higher suppression of in vitro SDF-1 release was observed for the hydrogel of Succ. Figure 2 shows the effect of hydrogel type on the SDF-1 release. The highest amount of SDF-1 remaining was observed for the Succ hydrogel.

\subsection{In Vivo Profiles of SDF-1 Release and Gelatin Hydrogel Degradation}

Figure 3 shows the time profiles of in vivo radioactivity remaining after implantation of Succ gelatin hydrogels incorporating ${ }^{125}$ I-labeled SDF-1 with different water contents. For the hydrogel incorporating ${ }^{125}$ I-labeled SDF-1, the radioactivity was retained for longer time periods than the solution of ${ }^{125} \mathrm{I}$-labeled SDF-1. The radioactivity of every hydrogel decreased with the implantation time. The gelatin 


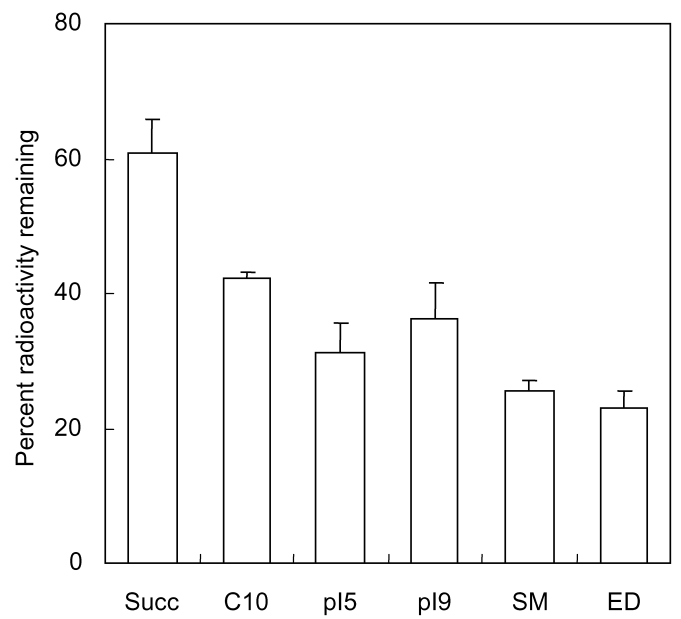

Figure 2. Percent radioactivity remaining of gelatin hydrogels incorporating ${ }^{125}$ I-labeled SDF-1 $24 \mathrm{~h}$ after subcutaneous implantation. Abbreviations on the $x$-axis are as given in the legend to Fig. 1.

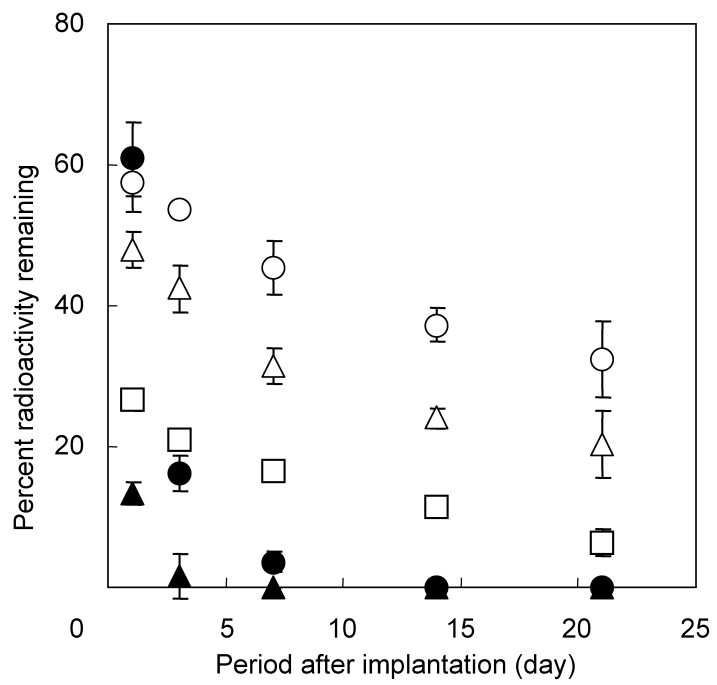

Figure 3. Time profiles of radioactivity remaining after subcutaneous implantation of ${ }^{125}$ I-labeled SDF-1-incorporating gelatin hydrogels with different water contents of $95.8(\bigcirc), 96.3(\triangle), 97.9(\square)$ and $98.8 \mathrm{wt} \%(\bullet)$ or subcutaneous injection of ${ }^{125}$ I-labeled SDF-1 solution ( $\left.\mathbf{\Delta}\right)$.

hydrogels with higher water content released SDF-1 faster than those with lower water content. Figure 4 shows the time profiles of in vivo radioactivity remaining after implantation of ${ }^{125}$ I-labeled gelatin hydrogels with different water contents. Similar to Fig. 3, the gelatin hydrogels with higher water contents degraded faster than those with lower water contents. Figure 5 shows the correlation of radioactivity remaining after implantation between the gelatin hydrogels incorporating 


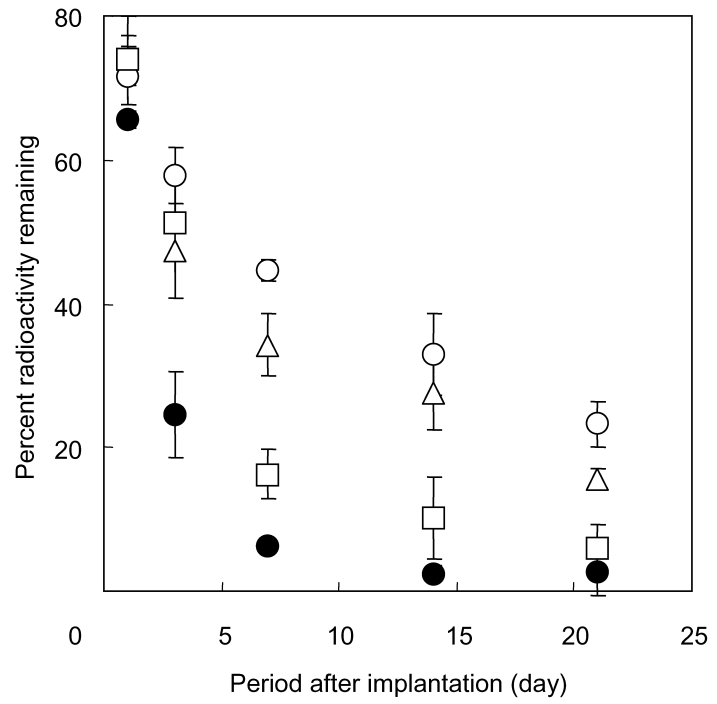

Figure 4. Time profiles of radioactivity remaining after subcutaneous implantation of ${ }^{125}$ I-labeled gelatin hydrogels with different water contents of $95.8(\bigcirc), 96.3(\triangle), 97.9(\square)$ and 98.8 wt\% (๑).

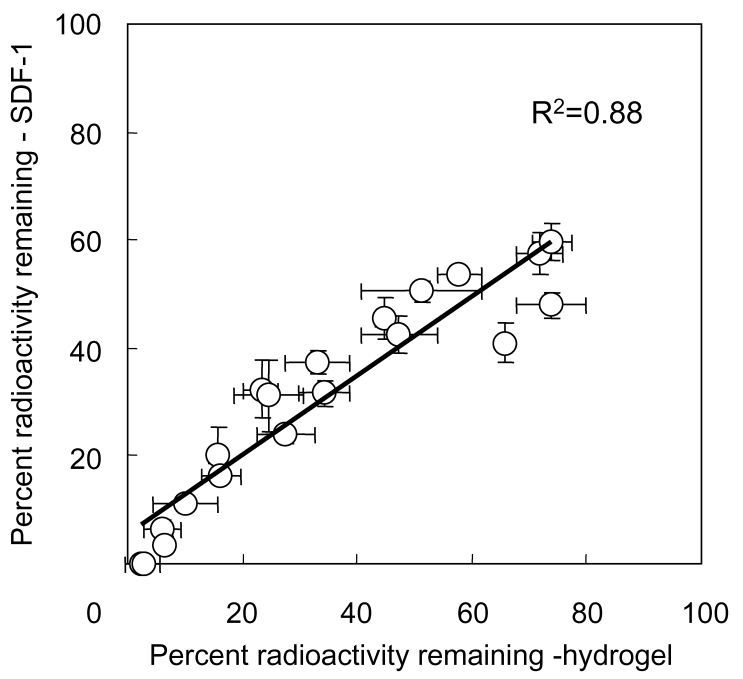

Figure 5. Relationship of remaining radioactivity after subcutaneous implantation into the mouse back between ${ }^{125}$ I-labeled SDF-1 incorporated in gelatin hydrogels and ${ }^{125}$ I-labeled hydrogels of release carrier.

${ }^{125}$ I-labeled SDF-1 and ${ }^{125}$ I-labeled gelatin hydrogels. The time profile of SDF-1 remaining was in good accordance with that of hydrogel remaining.

\subsection{Angiogenesis by Gelatin Hydrogels Incorporating SDF-1}

Figure 6 shows the mRNA expression of CXCR4, the receptor of SDF-1, around the implanted sites of gelatin hydrogels incorporating various amounts of SDF-1. The 


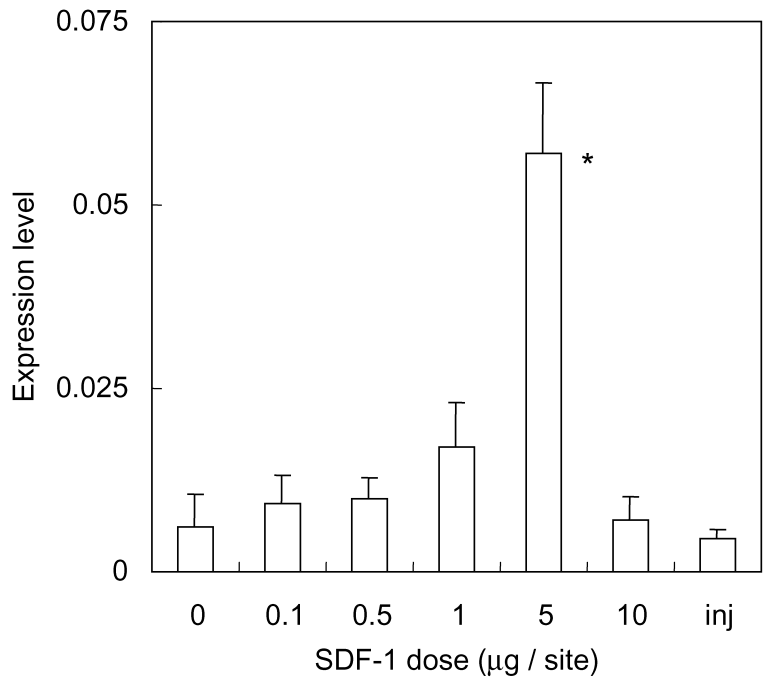

Figure 6. CXCR4 mRNA expression at the implanted site of gelatin hydrogels (water content $98.8 \mathrm{wt} \%$ ) incorporating various amounts of SDF-1 3 days after implantation. As control, mRNA expression was examined after injection of SDF-1 solution $(5 \mu \mathrm{g})$ (inj). ${ }^{*} P<0.05$, significant against the expression level of other groups.

expression level was up-regulated with the dose of SDF-1 implanted in the range of low doses. The level was significantly high at the dose of $5 \mu \mathrm{g} / \mathrm{site}$ compared with that of other doses.

Figure 7 shows the light microscopic photographs of the tissue site 3 days after implantation of gelatin hydrogel incorporating SDF-1. Figure 8 shows the time profiles of the number of blood vessel capillaries newly formed at the tissue site after implantation of gelatin hydrogels incorporating SDF-1. Significantly larger number of capillaries was observed at the site implanted with the hydrogel incorporating SDF-1 4 and 7 days after implantation than the case of the SDF-1 injection group. However, no significance in the capillary number was observed among all the experimental groups 10 days after treatment.

\section{Discussion}

This study clearly demonstrates that the controlled release of SDF-1 could be achieved by gelatin hydrogels. The SDF-1 release was experimentally confirmed to be effective in enhancing SDF-1-induced angiogenesis. The Succ hydrogel was suitable for SDF-1 release. The SDF-1 release enhanced the in vivo angiogenic activity to a significantly great extent compared with the SDF-1 solution.

SDF-1 is of positive charge at physiological $\mathrm{pH}(\mathrm{pH}$ 7.4) because of its IEP of 10.26. It is possible that SDF-1 molecules can electrostatically interact with the succinylated gelatin of negative charge, resulting in the suppressed SDF-1 release from the succinylated hydrogel accompanied with biodegradation. This hydrogel 


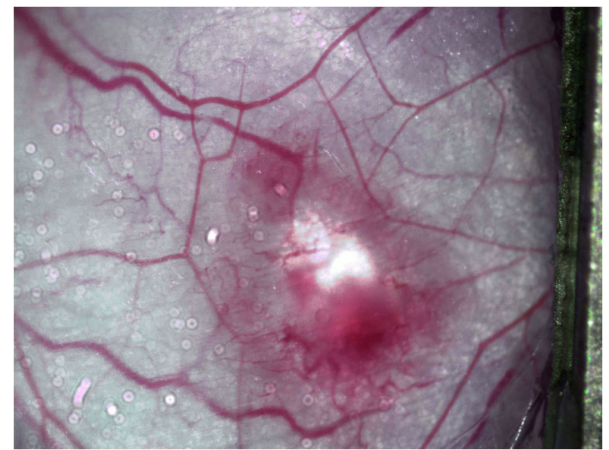

(a)

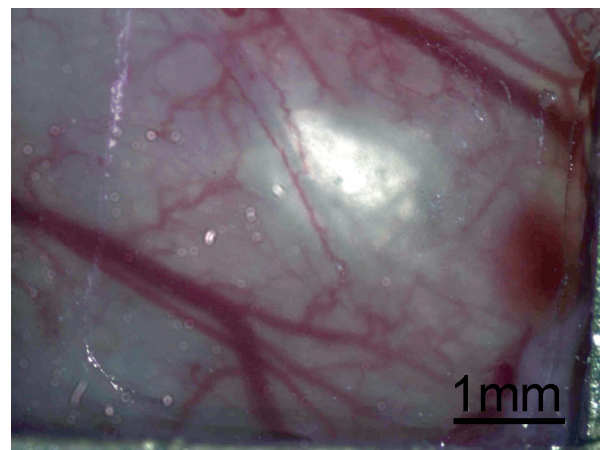

(c)

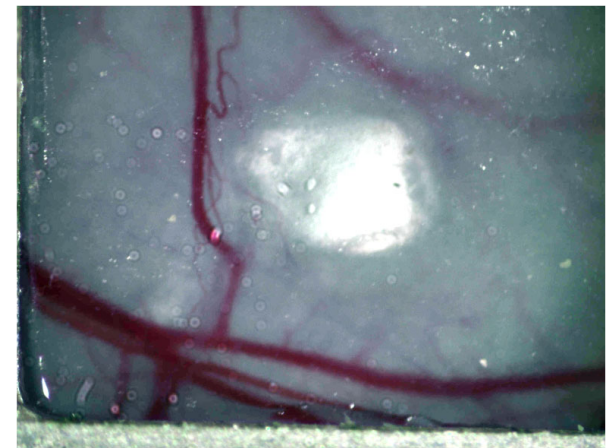

(b)

Figure 7. Light microscopic photographs of tissue site 3 days after implantation of gelatin hydrogel (water content $98.8 \mathrm{wt} \%$ ) incorporating $5 \mu \mathrm{g}$ SDF-1 (a) and empty gelatin hydrogel (b) or injection of SDF-1 solution $(5 \mu \mathrm{g})(\mathrm{c})$. Scale bar $=1 \mathrm{~mm}$. This figure is published in colour in the online edition of this journal that can be accessed via http://www.brill.nl/jbs

degradation-based SDF-1 release is experimentally confirmed by the in vitro and in vivo tests of SDF-1 release (Figs 1 and 2). Irrespective of the hydrogel type, the amount of SDF-1 released in vitro from gelatin hydrogels increased with time to attain a certain level and thereafter saturated. In this release system, the SDF-1 molecules are immobilized in the hydrogel through their physicochemical interaction with the hydrogel gelatin. Without the enzymatic degradation of hydrogels to generate water-soluble gelatin fragments, SDF-1 molecules immobilized are not released from the hydrogels. Based on this release mechanism, not all the SDF-1 molecules are released under the in vitro conditions in the absence of enzymes where the hydrogel is not degraded. The initial release of SDF-1 is due to the simple diffusion of free SDF-1 non-immobilized in the hydrogel. This release profile was identical with that of other growth factors reported previously $[9,15]$. The SDF-1-gelatin interaction would suppress the SDF-1 release from the hydrogel under the in vitro condition where the hydrogel is not degraded. Such a suppressed effect, although low, was observed for other types of hydrogels under the in vitro condition where the hydrogel is not degraded. This can be explained by different 


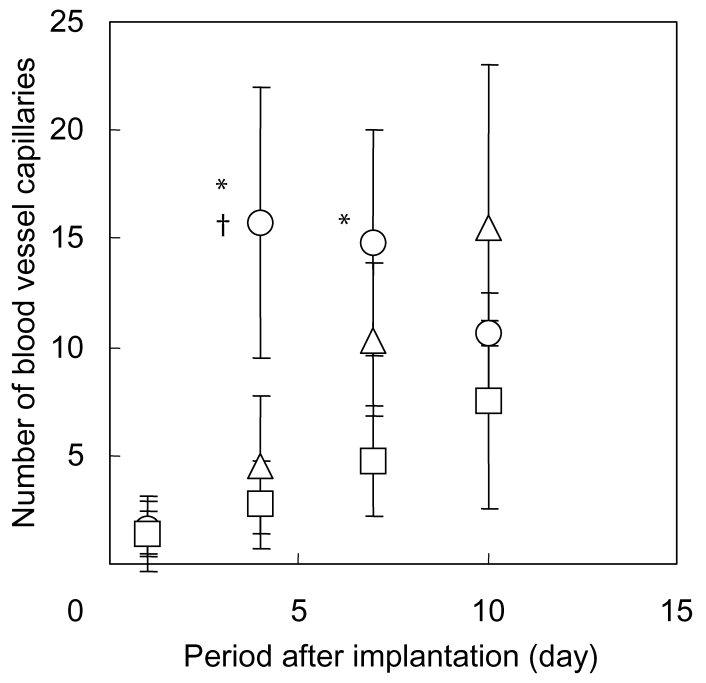

Figure 8. The number of blood vessel capillaries newly formed at the tissue site at different time intervals after implantation of gelatin hydrogels (water content $98.8 \mathrm{wt} \%$ ) incorporating 5 of SDF-1 $(\bigcirc)$ and empty gelatin hydrogels $(\triangle)$ or injection of SDF-1 solution $(5 \mu \mathrm{g})(\square) .{ }^{*} P<0.05$, significant against the capillary number of SDF-1 solution group, ${ }^{\dagger} P<0.05$, significant against the capillary number of empty gelatin hydrogel group.

interaction forces between SDF-1 and gelatin molecules. In addition to the electrostatic interaction there are hydrophobic and hydrogen bonding interactions between SDF-1 and gelatin molecules. In the case of basic fibroblast growth factor (bFGF) of positive charge, the electrostatic interaction mainly plays an important role in the bFGF immobilization into the hydrogel for the release [15]. Since the SDF-1 molecule has an electric nature similar to bFGF, it is conceivable that the electrostatic interaction with gelatin is prominent for the SDF-1 release. The in vitro (Fig. 1) and in vivo (Fig. 2) conditions affect the release profile of SDF-1. In the former, since PBS does not contain any enzymes to degrade the hydrogel, only the SDF-1 molecules in the free form can be released from the hydrogel. On the other hand, in the in vivo system, there are many enzymes and proteins. It is likely that upon degrading the hydrogel, the SDF-1 immobilized can be solubilized in water, resulting in the release from the hydrogel. Generally considering that the longer SDF-1 release results in the higher angiogenesis in vivo, in this study it is practically reasonable to take the hydrogel system which can release in vivo for longer time periods. Based on this point, the succinylated gelatin hydrogel was used for in vivo angiogenic experiment. The cross-linking extent of succinylated gelatin hydrogels was changed by the preparation conditions (Table 3 ) and the time profiles of SDF-1 release and hydrogels degradation were greatly influenced by the crosslinking extent of hydrogels (Figs 3 and 4). When estimated the cross-link density of hydrogels prepared (average water content 95.8, 96.3, 97.9 and $98.8 \mathrm{wt} \%$ ) based on the Flory swelling equation [16] was $58,42,11$ and $3.2 \times 10^{-7} / \mathrm{cm}^{3}$, respectively. 
On the other hand, the molecular size of SDF-1 dimer is reported to be smaller than $7.1 \mathrm{~nm}$ in diameter [17]. Since the size is small enough compared with the network of hydrogels calculated from the cross-linking density, we can say that a free SDF-1 molecule can easily pass through the water phase in hydrogel. Taken together, if the SDF-1 does not interact with the succinylated gelatin, it would be rapidly released from the hydrogel. When the percent remaining of SDF-1 was compared, it tended to become higher with a decrease in the water content of hydrogels. This finding can be explained in terms of cross-linking extent by glutaraldehyde. The cross-linking reaction decreased the amino groups of gelatin and, consequently, the $\mathrm{COOH} / \mathrm{NH}_{2}$ ratio of gelatin would become larger. It is possible that the increased negative charge increase the interaction degree between the SDF-1 and gelatin, resulting in increased percent remaining of SDF-1.

A good correlation of the in vivo time profile between the SDF-1 release and hydrogel degradation (Fig. 5) clearly indicates that the SDF-1 release was not governed by the simple diffusion mechanism of SDF-1, but by the degradation of gelatin hydrogel. This phenomenon was identical with that of bFGF release reported previously [18]. Taken together, we could be confirmed that SDF-1 was released by weakening electrostatic interaction following release carrier degradation.

CXCR4 is an inherent receptor of SDF-1, which is expressed on hematopoietic cells, vascular endothelial cells and mesenchymal stem cells [6, 19-24]. In Fig. 6, the mRNA expression around the implantation site of gelatin hydrogels incorporating SDF-1 was increased. The expression level compared with that of the positive control (thymus tissue) was depended on the SDF-1 implantation dose and the highest expression was observed at the dose of $5 \mu \mathrm{g}$. Taken together, it is highly possible that the implantation of SDF-1-incorporated gelatin hydrogels enhanced the accumulation of CXCR4 positive cells around the site implanted, resulting in the enhanced formation of capillary (Figs 7 and 8). Salcedo et al. [25] reported that angiogenesis was detected in mice after the local daily subcutaneous injection of $1 \mu \mathrm{g}$ SDF-1 four times. However, the effective threshold dose of SDF-1 to induce angiogenesis is still unknown. There are other reports that 60 or $100 \mathrm{ng}$ of SDF-1 dose was good for cell accumulation [26] or treatment of myocardiac infarction [27]. Further experiments are needed to determine the optimal dose of SDF-1 implanted. In the range of lower SDF-1 doses, the level of CXCR4-positive cells accumulated around the implanted site decreased with the dose, but higher SDF-1 doses decreased the level (Fig. 6). This phenomenon can be explained in terms of the SDF-1 receptor down-regulation. Generally, the chemokine action decreased when the dose is too high because of the down-regulation of the receptor [28]. An appropriate amount of SDF-1 released from the hydrogel would be effective in the mobilization of CXCR4-positive cells and consequently angiogenesis enhancement.

The in vivo angiogenic assay showed that faster and stronger formation of new capillaries was detected for the hydrogel incorporating SDF-1 than for the empty hydrogel and SDF-1 solution. We used succinylated gelatin hydrogel incorporating 
SDF-1 for the angiogenic assay in terms of positive cell accumulation of the receptor, CXCR4, because the maximum amount of SDF-1 remaining was observed after subcutaneous implantation with the hydrogel. It is possible that the gelatin hydrogel enabled SDF-1 to be released locally in a controlled manner, resulting in an enhanced recruitment of cells for angiogenesis (Figs 7 and 8). A significantly higher number of capillaries formed newly was observed for the gelatin incorporating SDF-1 4 and 7 days after implantation than in the case of the SDF-1 injection group. In addition, the mean number of capillaries seems to decrease for the gelatin incorporating SDF-1 10 days after treatment. However, no significanct difference in the capillary number was observed among all the experimental groups 10 days after treatment. These phenomena were also observed in the study previously reported from Ichioka et al. [14]. It is known that necessary mediators are secreted to initiate and promote the wound repair process at normal murine skin after creation of defect. SDF-1 is also reported to express in the inflammation stage [29, 30]. It could be thought that the normal wound repair process was accelerated by the implantation of gelatin hydrogels incorporating SDF-1. We believe that this strongly indicates feasibilities of the SDF-1-incorporated hydrogel in angiogenic induction. Many researchers have been reported on angiogenesis using vascular endothelial growth factor, FGF family, and other growth factors or chemokines [31-33]. It has been known that SDF-1 is induced by factors such as VEGF or bFGF, and plays an important role as a factor to recruit bone marrow-derived circulating cells [25, 34]. Considering the inherent nature of SDF-1, it is no doubt that the controlled release of SDF-1 is a promising strategy to induce angiogenesis by making use of the natural healing potential of the living body.

There are several reports about the controlled release of SDF-1. Schantz et al. [26] examined the homing of mesenchymal stem cells by SDF-1 treatment with an infusion pump in vivo. The combination of vascular endothelial growth factor, SDF-1, and bone morphogenetic protein-6 (60 ng each/10 days infusion) was effective in inducing vascularization into a poly(caprolactone) scaffold. Zhang et al. [27] reported the controlled release of SDF-1 from poly(ethylene glycol)-conjugated fibrin and the therapeutic effect with a myocardial infarction model. SDF-1 could be released from the fibrin matrix up to 10 days and the SDF-1 release could improve the symptom of acute myocardial infarction through an enhanced homing of c-kit ${ }^{+}$ cells. Hiasa et al. reported the gene expression of SDF-1 by an electroporation method with the plasmid DNA at the site of myocardiac infarction [35]. The SDF-1 protein was expressed up to 14 days in the muscle and the good therapeutic efficacy resulted from the mobilization of endothelial progenitor cells into the infarction site. The effective use of SDF-1 enhances the recruitment of key cells into the site necessary, resulting in the cells-based enhancement of angiogenesis thereat. To this end, it is necessary to develop a system for enhanced biological activity of SDF-1 in vivo. For tissue engineering and regenerative medicine therapy, minimally invasive and low-risk treatments should be required and developed. For our release system, only a single implantation of biodegradable hydrogel could enhance the natural 
healing potential of the body, and consequently assist positively the healing process of diseases. The release profiles of SDF-1 could be easily modified by changing the cross-linking extent of the hydrogels (Fig. 4). The SDF-1 release technology will be available to treat other diseases. The combination with other therapeutic agents is another strategy to enhance the therapeutic efficacy of tissue regeneration.

\section{References}

1. R. Langer, Tissue Eng. 13, 1 (2007).

2. Y. Kimura and Y. Tabata, J. Dermatol. Sci. 47, 189 (2007).

3. A. Marui, Y. Tabata, S. Kojima, M. Yamamoto, K. Tambara, T. Nishina, Y. Saji, K. Inui, T. Hashida, S. Yokoyama, R. Onodera, T. Ikeda, M. Fukushima and M. Komeda, Circ. J. 71, 1181 (2007).

4. M. Z. Ratajczak, E. Zuba-Surma, M. Kucia, R. Reca, W. Wojakowski and J. Ratajczak, Leukemia 20, 1915 (2006).

5. O. Kollet, A. Dar and T. Lapidot, Annu. Rev. Immunol. 25, 51 (2006).

6. T. Lapidot, A. Dar and O. Kollet, Blood 106, 1901 (2005).

7. R. S. Taichman, C. Cooper, E. T. Keller, K. J. Pienta, N. S. Taichman and L. K. McCauley, Cancer Res. 62, 1832 (2002).

8. D. K. Jin, K. Shido, H. G. Kopp, I. Petit, S. V. Shmelkov, L. M. Young, A. T. Hooper, H. Amano, S. T. Avecilla, B. Heissig, K. Hattori, F. Zhang, D. J. Hicklin, Y. Wu, Z. Zhu, A. Dunn, H. Salari, Z. Werb, N. R. Hackett, R. G. Crystal, D. Lyden and S. Rafii, Nature Med. 12, 557 (2006).

9. K. Hori, C. Sotozono, J. Hamuro, K. Yamasaki, Y. Kimura, M. Ozeki, Y. Tabata and S. Kinoshita, J. Control. Rel. 118, 169 (2007).

10. PAGI, PAGI (Photographic and Gelatin Industries) Method, 9th edn. PAGI, Tokyo (2002).

11. Y. Tabata, A. Nagano, M. Muniruzzaman and Y. Ikada, Biomaterials 19, 1781 (1998).

12. M. Ozeki and Y. Tabata, J. Biomater. Sci. Polym. Ed. 16, 549 (2005).

13. A. E. Bolton and W. M. Hunter, Biochem. J. 133, 529 (1973).

14. S. Ichioka, S. Kouraba, N. Sekiya, N. Ohura and T. Nakatsuka, Br. J. Plast. Surg. 58, 1124 (2005).

15. Y. Ikada and Y. Tabata, Adv. Drug. Deliv. Rev. 31, 287 (1998).

16. H. Park, K. Park and W. Shalaby, Biodegradable Hydrogels for Drug Delivery. Technomic, Lancaster, PA (1993).

17. E. K. Ryu, T. G. Kim, T. H. Kwon, I. D. Jung, D. Ryu, Y. M. Park, J. Kim, K. H. Ahn and C. Ban, Proteins 67, 1193 (2007).

18. Y. Tabata and Y. Ikada, Biomaterials 20, 2169 (1999).

19. C. C. Bleul, L. Wu, J. A. Hoxie, T. A. Springer and C. R. Mackay, Proc. Natl. Acad. Sci. USA 94, 1925 (1997).

20. S. K. Gupta, P. G. Lysko, K. Pillarisetti, E. Ohlstein and J. M. Stadel, J. Biol. Chem. 273, 4282 (1998).

21. R. Horuk, Cytokine Growth Factor Rev. 12, 313 (2001).

22. D. Y. Jo, S. Rafii, T. Hamada and M. A. Moore, J. Clin. Invest. 105, 101 (2000).

23. A. Kortesidis, A. Zannettino, S. Isenmann, S. Shi, T. Lapidot and S. Gronthos, Blood 105, 3793 (2005).

24. R. Mohle, F. Bautz, S. Rafii, M. A. Moore, W. Brugger and L. Kanz, Blood 91, 4523 (1998).

25. R. Salcedo, K. Wasserman, H. A. Young, M. C. Grimm, O. M. Howard, M. R. Anver, H. K. Kleinman, W. J. Murphy and J. J. Oppenheim, Am. J. Pathol. 154, 1125 (1999). 
26. J. T. Schantz, H. Chim and M. Whiteman, Tissue Eng. 13, 2615 (2007).

27. G. Zhang, Y. Nakamura, X. Wang, Q. Hu, L. J. Suggs and J. Zhang, Tissue Eng. 13, 2063 (2007).

28. A. Pelchen-Matthews, N. Signoret, P. J. Klasse, A. Fraile-Ramos and M. Marsh, Immunol. Rev. 168, 33 (1999).

29. A. M. Havens, E. Chiu, M. Taba, J. Wang, Y. Shiozawa, Y. Jung, L. S. Taichman, N. J. D'Silva, R. Gopalakrishnan, C. Wang, W. V. Giannobile and R. S. Taichman, J. Periodontol. 79, 845 (2008).

30. H. W. Jiang, J. Q. Ling and Q. M. Gong, J. Endodontol. 34, 1351 (2008).

31. F. Bussolino, A. Albini, G. Camussi, M. Presta, G. Viglietto, M. Ziche and G. Persico, Eur. J. Cancer 32A, 2401 (1996).

32. J. Folkman, Nature Rev. Drug Discov. 6, 273 (2007).

33. Y. Tabata, Drug Discov. Today 10, 1639 (2005).

34. M. Grunewald, I. Avraham, Y. Dor, E. Bachar-Lustig, A. Itin, S. Yung, S. Chimenti, L. Landsman, R. Abramovitch and E. Keshet, Cell 124, 175 (2006).

35. K. Hiasa, M. Ishibashi, K. Ohtani, S. Inoue, Q. Zhao, S. Kitamoto, M. Sata, T. Ichiki, A. Takeshita and K. Egashira, Circulation 109, 2454 (2004). 
Copyright of Journal of Biomaterials Science -- Polymer Edition is the property of VSP International Science Publishers and its content may not be copied or emailed to multiple sites or posted to a listserv without the copyright holder's express written permission. However, users may print, download, or email articles for individual use. 